\title{
A survey on structured deformations
}

\author{
M. Baía \\ CAMGSD and Departamento de Matemática \\ Instituto Superior Técnico, Av. Rovisco Pais, 1049-001, Lisboa, Portugal
}

\section{J. Matias}

CAMGSD and Departamento de Matemática Instituto Superior Técnico, Av. Rovisco Pais, 1049-001, Lisboa, Portugal

\section{P. M. Santos}

CAMGSD and Departamento de Matemática

Instituto Superior Técnico, Av. Rovisco Pais, 1049-001, Lisboa, Portugal

Dedicated to Waldyr M. Oliva on the occasion of his 80 th birthday

\begin{abstract}
In this work we briefly describe the theory of (first-order) structured deformations of continua as well as the variational problems arising from this theory.
\end{abstract}

\section{Introduction}

The theory of (first-order) structured deformations introduced by Del Piero \& Owen [16] sets a basis to address a large variety of problems in continuum mechanics where geometrical changes can be associated to smoothclassical deformations, piecewise deformations and more complex deformations for which an analysis at macroscopic and microscopic level is required. For instance, in a solid with a crystalline defective structure, separation of cracks at the macroscopic level may compete with slips and lattice distortions at the microscopic level preventing the use of classical theories where deformations are assumed to be smooth. The objective of the theory of structured deformations is to generalize the theoretical apparatus of continuum mechanics as a starting point for a unified description of bodies with

2000 Mathematics Subject Classification. 4E15, 49J45, 28A33, 46T30.

Key words: Structured Deformations, Relaxation, Functions of Bounded Variation. 
microstructure. It also turns out to be relevant to describe phenomena as plasticity, damage, creation of voids, mixing, and fracture in terms of the underlying microstructure.

From a variational point of view, the problem of assigning a free energy to a body that undergoes a structured deformation was first studied by Choksi $\&$ Fonseca [12] in the context of functions of special bounded variation, shortly denoted by SBV (we refer to Section 3 for the main notations and concepts used throughout this work). This energy was defined as the more effective way to build up the deformation using sequences of approximating simple deformations in SBV. The results obtained in [12] show that the bulk density of the free energy of a structured deformation can be influenced by the bulk and interfacial density of the free energy of these approximating sequences providing precise relations among them.

The overall plan of this work in the ensuing sections will be as follows: in Section 2 we briefly describe the theory of structured deformations according to [16]. In addition we present some examples and a generalization to second order structured deformations derived by Owen \& Paroni [25]. The purpose of Section 3 is to fix some notation and to give a brief overview of the concepts and main results that are used in Section 4. In this last section we discuss the notion of structured deformations in the context of SBV functions as defined in [12]. In addition we present the main result in [12] and a further generalization of this result to the BV-setting that was derived by Baía, Matias \& Santos[10].

\section{Structured deformations}

The main objective of the theory of structured deformations introduced in [16] (see also Del Piero \& Owen [17] and Owen [24]) is to describe how a continuous body with microstructure will deform under prescribed applied forces. An essential initial step towards this objective is that of defining this class of deformations. To this purpose, we start by introducing the classes of regions that bodies can occupy in the Euclidean space, identified in the sequel by $\mathbb{R}^{N}$ :

- An open and bounded set $\mathcal{A} \subset \mathbb{R}^{N}$ is said to be a fit region if it is of finite perimeter and its boundary has zero volume.

- A piecewise fit region is a finite union of fit regions.

The notion of structured deformation relies on the concepts of classical deformation and simple deformation:

- A classical deformation from a fit region $\mathcal{A}$ is an orientation preserving mapping $u: \mathcal{A} \rightarrow \mathbb{R}^{N}$ that extends to all of $\mathbb{R}^{N}$ as a $C^{1}$-function and that, in addition, is invertible with inverse of class $C^{1}$. 
- A simple deformation from a piecewise fit region $\mathcal{A}$ is a pair $(\mathcal{K}, u)$, where $\mathcal{K} \subset \mathcal{A}$ has zero volume and $u: \mathcal{A} \backslash K \rightarrow \mathbb{R}^{N}$ is injective and piecewise classical. The mapping $u$ and the set $\mathcal{K}$, where jump discontinuities are allowed, are called, respectively, the transplacement and the disarrangement site for the simple deformation $(\mathcal{K}, u)$.

In general, a simple deformation can separate a body, partially or entirely, into pieces and can deform each piece separately. These pieces can come in contact after deformation, but, because $u$ is injective, cannot interpenetrate. Each simple deformation is intended to describe macroscopic, geometrical changes in a body that arise when all or a portion of the body is disconnected through slip or separation. We remark that the set $\mathcal{K}$ was initially called in [16] the crack site in line with the interpretation of discontinuities in the continuum body as fractures. In this context, $\mathcal{K}$ can be seen basically as the collection of all points of the body at which a new crack is created and $u$ specifies the position occupied by the remaining points of the body after the deformation. We also observe that fit regions are not adequate to describe the concept of simple deformations since, for instance, still with reference to fractures, they exclude the existence of unopened cracks.

Structured deformations are a proper subset of a class of limits of sequences of simple deformations suitable to describe phenomena where discontinuities are allowed to diffuse through the body giving rise to the occurrence of structural changes at microscopic level. More precisely, a (firstorder) structured deformation $(\mathcal{K}, g, G)$ from a a piecewise fit region $\mathcal{A}$ consists of a simple deformation $(\mathcal{K}, g)$ from $\mathcal{A}$ and a continuous vector field $G: \mathcal{A} \backslash K \rightarrow \mathbb{R}^{N \times N}$ such that:

- $G$ is piecewise continuous in the closure of $\mathcal{A}$,

- there exists a positive number $m$ for which

$$
m \leq \operatorname{det} G(x) \leq \operatorname{det} \nabla g(x), \quad \forall x \in \mathcal{A} \backslash K .
$$

As proved in [16, Theorem 5.8] given a structured deformation from $\mathcal{A}$, $(\mathcal{K}, g, G)$, there exists a sequence of simple deformations from $\mathcal{A},\left\{\left(\mathcal{K}_{n}, u_{n}\right)\right\}$, such that

$$
u_{n} \stackrel{L^{\infty}}{\longrightarrow} g, \nabla u_{n} \stackrel{L^{\infty}}{\longrightarrow} G
$$

and

$$
\mathcal{K}=\bigcup_{p=1}^{\infty} \bigcap_{n=p}^{\infty} \mathcal{K}_{n}
$$

The site of all disarrangements (both macroscopic and microscopic) of a structured deformation from $\mathcal{A},(\mathcal{K}, g, G)$ is called its zone of disarrangements or fractured zone. It is given by the intersection over all sequences 
that determine $(\mathcal{K}, g, G)$ of all sets

$$
\bigcap_{p=1}^{\infty} \operatorname{clo}\left[\bigcup_{n=p}^{\infty} \mathcal{K}_{n}\right] \text {, }
$$

where the closure is taken relative to $\mathcal{A}$, and contains the set $\mathcal{K}$. We observe that the gradient $\nabla u_{n}$ of each transplacement of an approximating sequence $\left\{\left(\mathcal{K}_{n}, u_{n}\right)\right\}$ can be regarded as a local measure of deformation at those points of $\mathcal{A}$ at which no disarrangement or fracture occurs. Thus $G$ can be considered as a local measure of deformation without disarrangements or fracture, in the sense that $G$ is not affected by the presence of disarrangements or fractures at either the macroscopic and microscopic levels. It is also natural to consider $\nabla g$ as a local measure of the macroscopic deformation determined by the macroscopically observed transplacement $g$. For these reasons, $\nabla g$ and $G$ are called, respectively, the macroscopic deformation tensor and the tensor of deformation without disarrangements or fracture.

To illustrate these notions above, we present here two simple examples of structured deformations that can be found in [16].

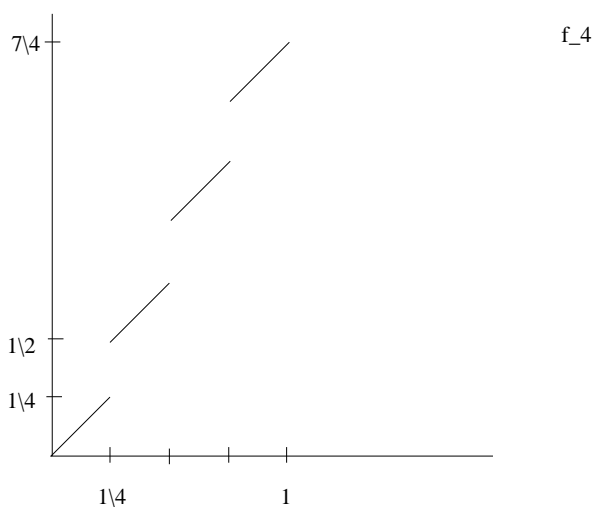

FiguRE 1. The broken ramp function $u_{n}$ for $n=4$

Example 2.1. (The broken ramp functions and its limit) Let $N=1$ and $\mathcal{A}=(0,1)$. For each $n \in \mathbb{N}$ let $\left(\mathcal{K}_{n}, u_{n}\right)$ be the simple deformation from $\mathcal{A}$ given by

$$
\mathcal{K}_{n}=\left\{\frac{1}{n}, \ldots, \frac{n-1}{n}\right\}
$$




$$
u_{n}(x):=x+\frac{k}{n}, \frac{k}{n} \leq x<\frac{k+1}{n}, k=0, \ldots, n-1
$$

(see Figure 1). It easily follows that

$$
u_{n} \underset{n \rightarrow \infty}{\stackrel{L^{\infty}(0,1)}{\longrightarrow}} g, \quad \nabla u_{n} \underset{n \rightarrow \infty}{\stackrel{L^{\infty}(0,1)}{\longrightarrow}} G
$$

where $g(x)=2 x$ and $G(x)=1$ and, in addition,

$$
\bigcup_{p=1}^{\infty} \bigcap_{n=p}^{\infty} \mathcal{K}_{n}=\emptyset \text {. }
$$

That is, $\left\{\left(\mathcal{K}_{n}, u_{n}\right)\right\}$ converges (in the sense above) to the structured deformation $(\mathcal{K}, g, G)$ where $\mathcal{K}=\emptyset$. In terms of the total distributional derivative we have that

$$
D u_{n}=1+\sum_{k=1}^{n-1} \frac{1}{n} \delta_{\frac{k}{n}},
$$

where $\delta_{a}$ stands for the Dirac mass at $x=a$, and $D u_{n} \rightarrow D g$ (in the sense of distributions). Thus, the part of D $u_{n}$ corresponding to jumps converges (in the sense of distributions) to $G-\nabla g$, which in this case, is the constant function 1. In other words, this example illustrate a typical situation in which each individual term of the determining sequence involves microfractures which, for $n$ large enough, spread all over the body with decreasing amplitudes of the associated jumps. In the limit the jumps disappear and $\mathcal{K}$ turns out to be the empty set (no macrofracture is present).

Example 2.2. (The deck of cards functions and its limit) Let $N=2$ and $\mathcal{A}=(0,1)^{2}$. For each $n \in \mathbb{N}$ let $\left(\mathcal{K}_{n}, u_{n}\right)$ be the simple deformation from $\mathcal{A}$ given by

$$
\begin{gathered}
\mathcal{K}_{n}=(0,1) \times\left\{\frac{k}{n}, k=1,2, \ldots, n-1\right\}, \\
u_{n}(x, y):=\left(x+d \frac{k}{n}, y\right), \frac{k-1}{n} \leq y<\frac{k}{n}, k=1, \ldots, n, d>0,
\end{gathered}
$$

(see Figure 2). In this case

$$
u_{n} \underset{n \rightarrow \infty}{\stackrel{L^{\infty}(0,1)}{\longrightarrow}} g, \quad \nabla u_{n} \underset{n \rightarrow \infty}{\stackrel{L^{\infty}(0,1)}{\longrightarrow}} G
$$

where $g(x, y)=(x+d y, y)$ and $G(x, y)=\mathbb{I}$ and, in addition,

$$
\bigcup_{p=1}^{\infty} \bigcap_{n=p}^{\infty} \mathcal{K}_{n}=\emptyset \text {. }
$$


f_8
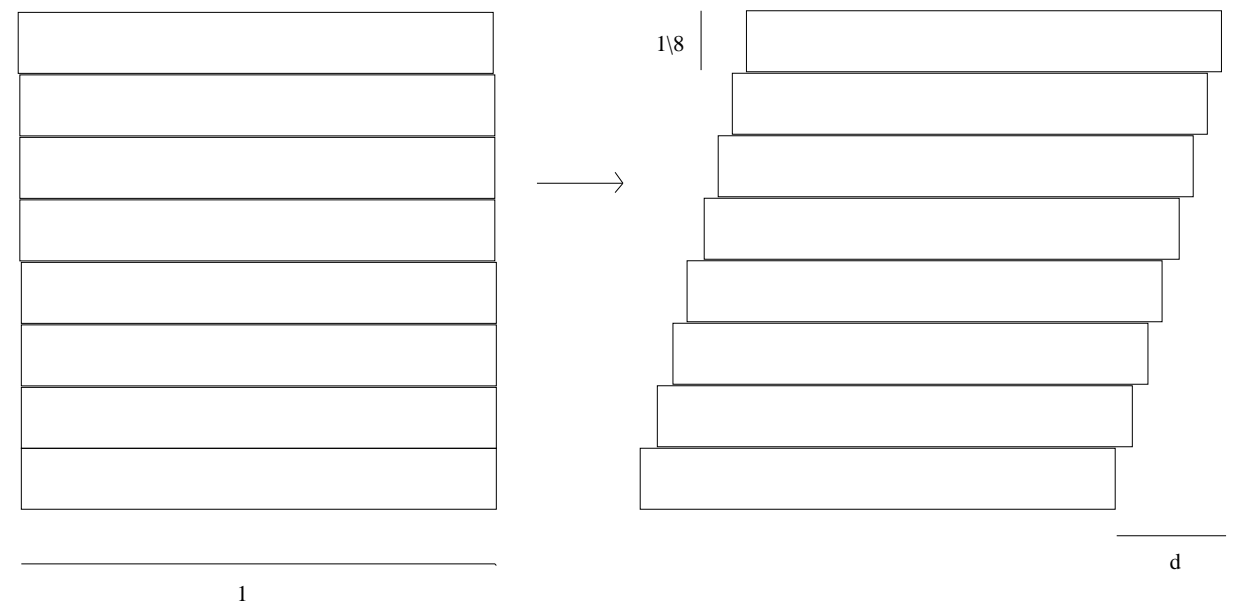

Figure 2. The deck of cards function $u_{n}$ for $n=8$
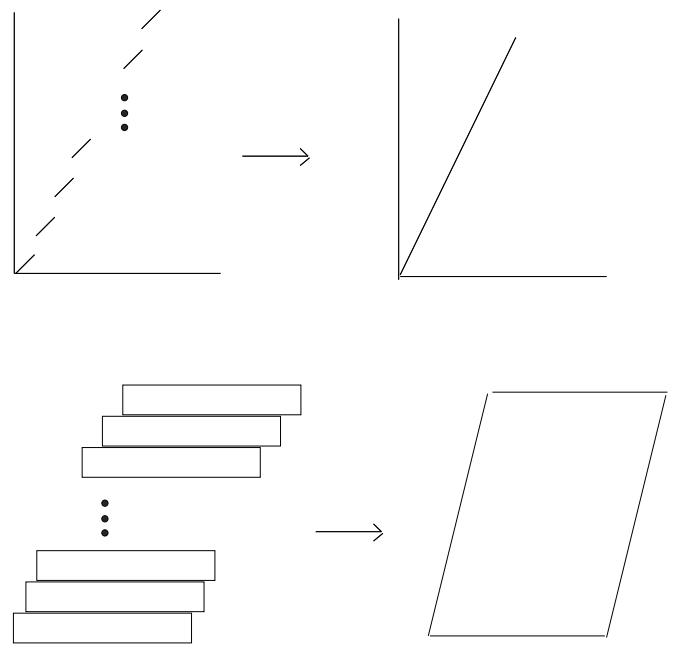

Figure 3. Convergence

That is, $\left\{\left(\mathcal{K}_{n}, u_{n}\right)\right\}$ converges to the structured deformation $(\emptyset, g, G)$ (see Figure 3). Thus, the macroscopic deformation is a simple shear, but the equality $G=\mathbb{I}$ means that there is no deformation without disarrangements.

São Paulo J.Math.Sci. 5, 2 (2011), 185-201 
In the context of of defective crystals the structured deformation $(\mathcal{K}, g, G)$ may therefore describe slips across the crystallographic planes, occurring at the microscopic level without deforming the lattice.

We finish this section by remarking that the principal concepts and results associated to first-order structured deformations were extended in Owen \& Paroni [25] through the notion of second-order structured deformation. This generalization allows for refinements of kinematical quantities such as acceleration and stretching which are not available through first-order structured deformations. Roughly speaking the original triple $(\mathcal{K}, g, G)$ is extended to a quadruple $(\mathcal{K}, g, G, H)$ where $H \in \mathbb{R}^{N \times N}$ is a piecewise continuous, second order symmetric tensor field. The principal result in [25] is an approximation theorem for second-order structured deformations that generalizes the corresponding result for first-order structured deformations and includes the effects of limits of second gradients and jumps in the first gradients of approximating deformations. A typical example of second-order structured deformation is the bending of a deck of cards (roughly speaking Figure 2 but bent). In this example, the corresponding second order structured deformation $(\emptyset, g, G, H)$ is such that $\nabla G \neq \nabla^{2} g \neq H$ and represents a macroscopic bending of a two-dimensional region with accompanying disarrangements due to jumps in both the approximating sequence $u_{n}$ and its gradient $\nabla u_{n}$.

\section{Preliminaries on BV functions}

In this section we briefly summarize some facts on functions of bounded variation which will be the natural framework for a mathematical theory on structured deformations. We refer to Ambrosio, Fusco and Pallara [5], Evans and Gariepy [18], Federer [19], Giusti [22] and Ziemer [27] for a detailed description of this subject. We will use throughout the following notations:

- $\mathbb{R}^{d \times N}$ will be identified with the space of real-valued $d \times N$ matrices, $N \geq 1, d \geq 1$,

- $\mathbb{R}^{d \times N \times N}$ is the set of real tensors of order $d \times N \times N$,

- $\Omega \subset \mathbb{R}^{N}$, will denote an open bounded set,

- $\mathcal{A}(\Omega)$ is the family of all open subsets of $\Omega$,

- $\mathcal{M}(\Omega)$ is the set of finite Radon measures on $\Omega$,

- $\mathcal{M}\left(\Omega ; \mathbb{R}^{d}\right)$ is the space of finite $\mathbb{R}^{d}$-valued Radon measures on $\Omega$,

- $\|\mu\|$ stands for the total variation of a measure $\mu \in \mathcal{M}(\Omega)$,

- $S^{N-1}$ stands for the unit sphere in $\mathbb{R}^{N}$,

- $\mathcal{L}^{N}$ denotes the Lebesgue measure in $\mathbb{R}^{N}$,

- $\mathcal{H}^{N-1}$ denotes the $N-1$ dimensional Hausdorff measure in $\mathbb{R}^{N}$, 
- $\mu\lfloor A$ denotes the restriction of the measure $\mu$ to the $\mu$-measurable set $A$, i.e. is the measure defined by $\mu\lfloor A(E)=\mu(A \cap E)$,

- $e_{i}$ denotes the $i^{\text {th }}$ element of the canonical basis of $\mathbb{R}^{N}$ for $i=$ $1, \ldots N$,

- $Q$ denotes the unit cube centered at the origin with one side orthogonal to $e_{N}$,

- $Q(x, \delta)$ denotes a cube centered at $x \in \Omega$ with side length $\delta$ and with one side orthogonal to $e_{N}$,

- $Q_{\nu}(x, \delta)$ is the cube centered at $x \in \Omega$ with side length $\delta$ and with one side orthogonal to $\nu \in S^{N-1}$,

- $Q_{\nu}:=Q_{\nu}(0,1)$,

- $a \otimes b$ denotes the tensorial product of vectors $a$ and $b$.

A function $u \in L^{1}\left(\Omega ; \mathbb{R}^{d}\right)$ is said to be of bounded variation, and we write $u \in B V\left(\Omega ; \mathbb{R}^{d}\right)$, if all its first distributional derivatives $D_{j} u_{i} \in \mathcal{M}(\Omega)$ for $i=1, \ldots, d$ and $j=1, \ldots, N$. The matrix-valued measure whose entries are $D_{j} u_{i}$ is denoted by $D u$. The space $B V\left(\Omega ; \mathbb{R}^{d}\right)$ is a Banach space when endowed with the norm

$$
\|u\|_{B V}=\|u\|_{L^{1}}+\|D u\|(\Omega) .
$$

By the Lebesgue Decomposition Theorem $D u$ can be split into the sum of two mutually singular measures $D^{a} u$ and $D^{s} u$ (the absolutely continuous part and singular part, respectively, of $D u$ with respect to $\left.\mathcal{L}^{N}\right)$. By $\nabla u$ we denote the Radon-Nikodým derivative of $D^{a} u$ with respect to $\mathcal{L}^{N}$, so that we can write

$$
D u=\nabla u \mathcal{L}^{N}\left\lfloor\Omega+D^{s} u .\right.
$$

Let $\Omega_{u}$ be the set of points where the approximate limit of $u$ exists, i.e., $x \in \Omega$ such that there exist $z \in \mathbb{R}^{N}$ with

$$
\lim _{\varepsilon \rightarrow 0} f_{Q(x, \varepsilon)}|u(y)-z| d y=0 .
$$

If $x \in \Omega_{u}$ and $z=u(x)$ we say that $u$ is approximately continuous at $x$ (or that $x$ is a Lebesgue point of $u$ ). The function $u$ is approximately continuous $\mathcal{L}^{N}$-a.e. $x \in \Omega_{u}$ and

$$
\mathcal{L}^{N}\left(\Omega \backslash \Omega_{u}\right)=0 .
$$

Let $S_{u}$ be the jump set of this function, i.e., the set of points $x \in \Omega \backslash \Omega_{u}$ for which there exists $a, b \in \mathbb{R}^{N}$ and a unit vector $\nu \in S^{N-1}$, normal to $S_{u}$ 
at $x$, such that $a \neq b$ and

$$
\lim _{\varepsilon \rightarrow 0^{+}} \frac{1}{\varepsilon^{N}} \int_{\left\{y \in Q_{\nu}(x, \varepsilon):(y-x) \cdot \nu>0\right\}}|u(y)-a| d y=0
$$

and

$$
\lim _{\varepsilon \rightarrow 0^{+}} \frac{1}{\varepsilon^{N}} \int_{\left\{y \in Q_{\nu}(x, \varepsilon):(y-x) \cdot \nu<0\right\}}|u(y)-b| d y=0 .
$$

The triple $(a, b, \nu)$ uniquely determined by (3.2) and (3.3) up to permutation of $(a, b)$, and a change of sign of $\nu$ and is denoted by $\left(u^{+}(x), u^{-}(x), \nu_{u}(x)\right)$.

If $u \in B V(\Omega)$ it is well known that $S_{u}$ is countably $N-1$ rectifiable, i.e.

$$
S_{u}=\bigcup_{n=1}^{\infty} K_{n} \cup E
$$

where $\mathcal{H}^{N-1}(E)=0$ and $K_{n}$ are compact subsets of $C^{1}$-hypersurfaces. Furthermore, $\mathcal{H}^{N-1}\left(\left(\Omega \backslash \Omega_{u}\right) \backslash S_{u}\right)=0$ and the following decomposition holds

$$
D u=\nabla u \mathcal{L}^{N}\left\lfloor\Omega+[u] \otimes \nu_{u} \mathcal{H}^{N-1}\left\lfloor S_{u}+D^{c} u,\right.\right.
$$

where $[u]:=u^{+}-u^{-}$and $D^{c} u$ is the Cantor part of the measure $D u$, i.e., $D^{c} u=D^{s} u\left\lfloor\left(\Omega_{u}\right)\right.$.

If $\Omega$ is an open and bounded set with Lipschitz boundary then the outer unit normal to $\partial \Omega$ (denoted by $\nu$ ) exists $\mathcal{H}^{N-1}$ a.e. and the trace for functions in $B V\left(\Omega ; \mathbb{R}^{d}\right)$ is defined.

The following results on BV functions will be used in the sequel.

Lemma 3.1. Let $u \in B V\left(\Omega ; \mathbb{R}^{d}\right)$. There exist piecewise constant functions $u_{n}$ such that $u_{n} \rightarrow u$ in $L^{1}\left(\Omega ; \mathbb{R}^{d}\right)$ and

$$
\|D u\|(\Omega)=\lim _{n \rightarrow \infty}\left\|D u_{n}\right\|(\Omega)=\lim _{n \rightarrow \infty} \int_{S_{u_{n}}}\left|\left[u_{n}\right](x)\right| d H^{N-1}(x) .
$$

The space of special functions of bounded variation introduced by De Giorgi and Ambrosio [15] in problems arising from pattern recognition and 
the mathematical theory of liquid crystals, $S B V\left(\Omega ; \mathbb{R}^{d}\right)$, is the space of functions $u \in B V\left(\Omega ; \mathbb{R}^{d}\right)$ such that $D^{c} u=0$, i.e. for which

$$
D u=\nabla u \mathcal{L}^{N}+[u] \otimes \nu_{u} \mathcal{H}^{N-1}\left\lfloor S_{u} .\right.
$$

The next result is a Lusin type theorem for gradients due to Alberti [2] and it is essential for the proofs of the results presented on this survey.

Theorem 3.2. Given $f \in L^{1}\left(\Omega ; \mathbb{R}^{d \times N}\right)$ there exists $u \in S B V\left(\Omega ; \mathbb{R}^{d}\right)$ and a Borel function $g: \Omega \rightarrow \mathbb{R}^{d \times N}$ such that

$$
\begin{gathered}
D u=f \mathcal{L}^{N}+g \mathcal{H}^{N-1}\left\lfloor S_{u},\right. \\
\int_{S_{u}}|g| d \mathcal{H}^{N-1} \leq C\|f\|_{L^{1}\left(\Omega ; \mathbb{R}^{d \times N}\right)} .
\end{gathered}
$$

Remark 3.3. From the proof of Theorem 3.2 it follows also that

$$
\|u\|_{L^{1}(\Omega)} \leq C\|f\|_{L^{1}\left(\Omega ; \mathbb{R}^{d \times N}\right)} .
$$

Following Carriero, Leaci and Tomarelli (see [13] and [14]) we define,

$$
S B V^{2}\left(\Omega ; \mathbb{R}^{d}\right)=\left\{v \in S B V\left(\Omega ; \mathbb{R}^{d}\right), \nabla v \in S B V\left(\Omega ; \mathbb{R}^{d \times N}\right)\right\}
$$

If $u \in S B V^{2}\left(\Omega ; \mathbb{R}^{d}\right)$ we use the notation $\nabla^{2} u=\nabla(\nabla u)$, that is, $\nabla^{2} u$ is the absolutely continuous part of $D(\nabla u)$ with respect to Lebesgue measure. We will also denote by

$$
B V^{2}\left(\Omega ; \mathbb{R}^{d}\right)=\left\{v \in B V\left(\Omega ; \mathbb{R}^{d}\right), \nabla v \in B V\left(\Omega ; \mathbb{R}^{d \times N}\right)\right\} .
$$

\section{Free energies for structured deformations in the context of SBV functions. Generalization to the BV-setting}

The question of assigning a free energy to a body that undergoes a structured deformation was first studied by Choksi \& Fonseca [12] within a broader notion of structured deformation based on SBV functions. Our objective in this section is to present the work in [12] (see Subsection 4.1) as well as a further generalization to the BV-setting derived in Baía, Matias \& Santos [10] (see Subsection 4.2). 
4.1. SBV-setting. According to [12] a structured deformation in $\Omega$ is a pair $(g, G)$ where the macroscopic deformation $g$ is an element of $S B V\left(\Omega ; \mathbb{R}^{d}\right)$ and the part of the deformation without disarrangements $G$ is an integrable tensor field in $\Omega$ (the crack site is naturally identified with the jump set of $g$ ). The authors define the energy assigned to a structured deformation $(g, G)$ as the more effective way to build up the deformation using sequences of approximating simple deformations in SBV. We describe this process in two steps:

Step 1. It was first proved in [12] that given such a pair $(g, G)$ there exist deformations $u_{n} \in S B V\left(\Omega ; \mathbb{R}^{d}\right)$ with

$$
u_{n} \stackrel{L^{1}}{\longrightarrow} g \text { and } \nabla u_{n} \stackrel{\mathcal{M}\left(\Omega ; \mathbb{R}^{d \times N}\right)}{\longrightarrow} G
$$

(which provides the counterpart to the $S B V$-setting of the Approximation Theorem in [16] with respect to a weaker convergence). Indeed, given $u_{n}$ $\stackrel{L^{1}}{\longrightarrow} g$ by Theorem 3.2 there exist $h_{n} \in S B V\left(\Omega ; \mathbb{R}^{d}\right)$ such that $\nabla h_{n}=G-\nabla u_{n}$. On the other hand, using Lemma 3.1 it is possible to find for fixed $n$ a sequence $h_{n, m}$ of piecewise constant functions such that $h_{n, m} \underset{m \rightarrow \infty}{\stackrel{L^{1}}{\longrightarrow}} h_{n}$. Setting

$$
v_{n, m}:=u_{n}+h_{n}-h_{n, m}
$$

the conclusion now follows by a standard diagonalization argument.

Step 2. As a result of Step 1, the energy associated to $(g, G), I(g, G)$, was defined as the relaxation with respect to the topology given in (4.1) of the functional

$$
\mathcal{E}(u)=\int_{\Omega} \mathcal{W}(\nabla u) d x+\int_{S_{u}} \Psi\left([u], \nu_{u}\right) d \mathcal{H}^{N-1}, \quad u \in S B V\left(\Omega ; \mathbb{R}^{d}\right),
$$

for appropriate bulk and interfacial densities $\mathcal{W}$ and $\Psi$, that is

$$
I(g, G):=\inf _{\left\{u_{n}\right\} \subset S B V\left(\Omega ; \mathbb{R}^{d}\right)}\left\{\liminf _{n \rightarrow \infty} \mathcal{E}\left(u_{n}\right), \quad u_{n} \stackrel{L^{1}}{\longrightarrow} g, \quad \nabla u_{n} \stackrel{\mathcal{M}\left(\Omega ; \mathbb{R}^{d \times N}\right)}{\longrightarrow} G\right\} .
$$

The main objective in [12] was to characterize $I(g, G)$ through an integral representation formula. More precisely, the authors characterized different versions of $I$ according with the convergence arising from the hypotheses on the energy densities $\mathcal{W}$ and $\Psi$ that are of order technical and include coercivity and homogeneity of $\Psi$. As explained in [12] these hypotheses are not acceptable from the physical point of view and can be relaxed. We summarize the main results in [12] as follows. 
Case $p=1$. Let

$I_{0}(g, G):=$

$$
\begin{aligned}
& \inf _{\left\{u_{n}\right\} \subset S B V\left(\Omega ; \mathbb{R}^{d}\right)}\left\{\liminf _{n \rightarrow \infty} \mathcal{E}\left(u_{n}\right), u_{n} \stackrel{L^{1}}{\longrightarrow} g, \nabla u_{n} \stackrel{\mathcal{M}\left(\Omega ; \mathbb{R}^{d \times N}\right)}{\longrightarrow} m, \frac{d m}{d \mathcal{L}^{N}}=G\right\}, \\
& I_{1}(g, G):= \\
& \inf _{\left\{u_{n}\right\} \subset S B V\left(\Omega ; \mathbb{R}^{d}\right)}\left\{\liminf _{n \rightarrow \infty} \mathcal{E}\left(u_{n}\right), u_{n} \stackrel{L^{1}}{\longrightarrow} g, \nabla u_{n} \stackrel{\mathcal{M}\left(\Omega ; \mathbb{R}^{d \times N}\right)}{\longrightarrow} G\right\} .
\end{aligned}
$$

Under appropriate hypotheses it is proven that

$$
I_{0}(g, G)=\int_{\Omega} H_{1}(\nabla g(x), G(x)) d x+\mu_{s}(\Omega)
$$

for some Radon measure $\mu_{s}$, absolutely continuous with respect to $\mathcal{H}^{N-1}\left\lfloor S_{g}\right.$, and where for $A, B \in \mathbb{R}^{d \times N}$ we have that

$$
\begin{array}{r}
\inf _{\substack{u \in S B V\left(Q ; \mathbb{R}^{d}\right),|\nabla u| \in L^{1}(Q)}}^{H_{1}(A, B):=}\left\{\int_{Q} \mathcal{W}(\nabla u) d x+\int_{S_{u} \cap Q} \Psi([u], \nu) d \mathcal{H}^{N-1},\right. \\
u\left\lfloor_{\partial Q}=A x, \int_{Q} \nabla u d x=B\right\} .
\end{array}
$$

In addition

$$
I_{1}(g, G)=\int_{\Omega} H_{1}(\nabla g(x), G(x)) d x+\int_{S_{g} \cap \Omega} h_{1}\left([g], \nu_{g}\right) d \mathcal{H}^{N-1}
$$

where for $\lambda \in \mathbb{R}^{d}$ and $\nu \in S^{N-1}$

$$
\begin{aligned}
& h_{1}(\lambda, \nu):=\inf _{u \in S B V\left(Q ; \mathbb{R}^{d}\right)}\left\{\int_{Q_{\nu}} \mathcal{W}^{\infty}(\nabla u) d x+\int_{S_{u} \cap Q} \Psi\left([u], \nu_{u}\right) d \mathcal{H}^{N-1},\right. \\
& u\left\lfloor_{\partial Q_{\nu}}=u_{\lambda, \nu}, \quad \int_{Q_{\nu}} \nabla u d x=0\right\}
\end{aligned}
$$

being, as usual, the recession function of $\mathcal{W}$ defined by

$$
\mathcal{W}^{\infty}(A):=\limsup _{t \rightarrow \infty} \frac{W(t A)}{t}
$$


and

$$
u_{\lambda, \nu}(x):= \begin{cases}0 & \text { if } x \in Q_{\nu} \text { and }-\frac{1}{2} \leq x . \nu<0 \\ \lambda & \text { if } x \in Q_{\nu} \text { and } 0 \leq x . \nu<\frac{1}{2} .\end{cases}
$$

Case $p>1$. Let

$$
\begin{array}{r}
I_{p}(g, G):=\inf _{\left\{u_{n}\right\} \subset S B V\left(\Omega ; \mathbb{R}^{d}\right)}\left\{\liminf _{n \rightarrow \infty} \mathcal{E}\left(u_{n}\right), u_{n} \stackrel{L^{1}}{\longrightarrow} g, \nabla u_{n} \stackrel{\mathcal{M}\left(\Omega ; \mathbb{R}^{d \times N}\right)}{\longrightarrow} G,\right. \\
\left.\sup _{n}\left|\nabla u_{n}\right|_{L^{p}\left(\Omega ; \mathbb{R}^{d}\right)}<\infty\right\} .
\end{array}
$$

Then, under some additional hypotheses

$$
I_{p}(g, G)=\int_{\Omega} H_{p}(\nabla g(x), G(x)) d x+\int_{S_{g} \cap \Omega} h_{p}([g]) d \mathcal{H}^{N-1}
$$

where, for $A, B \in \mathbb{R}^{d \times N}$ and $\lambda \in \mathbb{R}^{d}$

$$
\begin{array}{r}
\inf _{\substack{u \in S B V\left(Q ; \mathbb{R}^{d}\right),|\nabla u| \in L^{1}(Q)}}^{H_{p}(A, B):=}\left\{\int_{Q} \mathcal{W}(\nabla u) d x+\int_{S_{u} \cap Q} \Psi([u], \nu) d \mathcal{H}^{N-1},\right. \\
u\left\lfloor_{\partial Q}=A x, \int_{Q} \nabla u d x=B\right\}
\end{array}
$$

and

$$
\begin{aligned}
& h_{p}(\lambda):= \\
& \inf _{u \in S B V\left(Q ; \mathbb{R}^{d}\right)}\left\{\int_{S_{u} \cap Q} \Psi([u], \nu) d \mathcal{H}^{N-1}, u\left\lfloor_{\partial Q}=u_{\lambda, e_{N}}, \nabla u=0 \mathcal{L}^{N}-\text { a.e. }\right\} .\right.
\end{aligned}
$$

As noticed in [12] the new bulk density $H_{p}$ is essentially the same for all $p \geq 1$ and it exhibits the interaction between the initial bulk and interfacial energies $\mathcal{W}$ and $\Psi$. In the case $p>1$, as admissible sequences are taken so that $\left\{\left|\nabla u_{n}\right|\right\}$ is bounded in $L^{p}$, then the new interfacial energy is independent from $\mathcal{W}$. Roughly speaking this means that, in these cases, it is cheaper to approximate jumps with jumps rather than with sharp gradients, and it is a contrast with the case $p=1$ where there is a contribution of $\mathcal{W}$, through its recession function, in $h_{1}$. 
We finish this part by remarking that the principal difference of the characterizations derived in [12] with respect to previous integral representation results for similar relaxed energies, where relaxation is taken with respect to the $L^{1}$ (BV weak) topology (cf. Ambrosio \& Dal Maso [4], Barroso, Bouchitté, Buttazo \& Fonseca [6], Braides \& Coscia [7], Bouchitté, Fonseca \& Mascarenhas [8], Bouchitté, Fonseca, Leoni \& Mascarenhas [9], Fonseca \& Müller [20,21]), is the fact that gradients of approximating sequences $\left\{u_{n}\right\}$ in (4.1) are constrained to converge to the given function $G$ (not necessarily $\nabla g$ ). In this case, if $\nabla u_{n} \rightarrow G$ in $L^{1}$, the difference $G-D g$ is achieved by the limit of singular measures since $D u_{n} \rightarrow D g$ in the sense of distributions. Moreover, the Hausdorff measure of the jump set of $u_{n}$ tends necessarily to infinity, otherwise Theorem 2.1 in Ambrosio [1] asserts that $G=\nabla g$ almost everywhere.

In the context of defective crystals, as already mentioned in [12], (4.2) can be interpreted as a way to realize the deformed crystal by piecing together elastic crystals at a finer and finer scale. We refer to Choksi, Del Piero, Fonseca \& Owen [11] where the framework introduced in [12] has been used to predict in simple models the origins and main characteristics of phenomena as fracture, yielding and histerisis, with applications to single defective crystals (see Section 4.3 in [11]).

4.2. BV-setting. The notions and results derived in [12] have been generalized to the full BV-setting for a class of second order energies suitable to the study of equilibrium configurations of thin defective crystalline structures in [10]. As pointed out in Matias \& Santos[23] the energy considered in [12] is not appropriate to this study since some control in the second order derivatives of the deformation is needed to avoid geometrical obstacles in the thin film limit.

To present the main result in [10] we start by introducing the space of generalized structured deformations

$$
G S D\left(\Omega ; \mathbb{R}^{d}\right):=B V^{2}\left(\Omega ; \mathbb{R}^{d}\right) \times B V\left(\Omega ; \mathbb{R}^{d \times N}\right)
$$

For any $(g, G) \in G S D\left(\Omega ; \mathbb{R}^{d}\right)$ we consider the relaxed energy

$$
I(g, G)=\inf _{\left\{u_{n}\right\} \subset S B V^{2}\left(\Omega ; \mathbb{R}^{d}\right)}\left\{\liminf _{n \rightarrow \infty} E\left(u_{n}\right), u_{n} \stackrel{L^{1}}{\longrightarrow} g, \nabla u_{n} \stackrel{L^{1}}{\longrightarrow} G\right\}
$$


where

$$
\begin{aligned}
E(u)=\int_{\Omega} W\left(\nabla u, \nabla^{2} u\right) d x & +\int_{S_{u}} \Psi_{1}([u], \\
& \left.\nu_{u}\right) d \mathcal{H}^{N-1} \\
& +\int_{S_{\nabla u}} \Psi_{2}\left([\nabla u], \nu_{\nabla u}\right) d \mathcal{H}^{N-1}
\end{aligned}
$$

for $u \in S B V^{2}\left(\Omega ; \mathbb{R}^{d}\right)$, and the functions $W, \Psi_{1}, \Psi_{2}$ satisfy hypotheses similar to the ones considered in [12]. The main result in this work asserts that there exist bulk and interfacial densities $W_{1}, W_{2}, \gamma_{1}, \gamma_{2}$ such that for all $(g, G) \in G S D\left(\Omega ; \mathbb{R}^{d}\right)$

$$
\begin{aligned}
I(g, G)= & \int_{\Omega}\left(W_{1}(G-\nabla g)+W_{2}(G, \nabla G)\right) d x+\int_{S_{g}} \gamma_{1}\left([g], \nu_{g}\right) d \mathcal{H}^{N-1} \\
& +\int_{S_{G}} \gamma_{2}\left(G^{+}, G^{-}, \nu_{G}\right) d \mathcal{H}^{N-1}+\int_{\Omega} W_{1}\left(-\frac{d D^{c} g}{d\left|D^{c} g\right|}\right) d\left|D^{c} g\right| \\
& +\int_{\Omega} W_{2}^{\infty}\left(G, \frac{d D^{c} G}{d\left|D^{c} G\right|}\right) d\left|D^{c} G\right|
\end{aligned}
$$

where $W_{2}^{\infty}$, as usual, denotes the recession function of $W_{2}$ in the second variable, that is,

$$
W_{2}^{\infty}(A, B)=\limsup _{t \rightarrow \infty} \frac{W(A, t B)}{t}, \quad A \in \mathbb{R}^{d \times N}, B \in \mathbb{R}^{d \times N \times N} .
$$

To show (4.4) a similar relaxation result in the $S B V$-setting is first derived. Althought this characterization could have been derived directly for the whole energy $I(g, G)$ using localization and blow up methods, Alberti's theorem (Theorem 3.2 above) allows the authors to divide this energy into two first order relaxed energies $I_{1}(g, G)$ and $I_{2}(G)$, rendering the arguments more concise. More precisely it was first proved that

$$
I(g, G)=I_{1}(g, G)+I_{2}(G)
$$

where

$$
\begin{array}{r}
I_{1}(g, G)=\inf _{\left\{u_{n}\right\} \subset S B V^{2}\left(\Omega ; \mathbb{R}^{d}\right)}\left\{\liminf _{n \rightarrow \infty} \int_{S_{u_{n}}} \Psi_{1}\left(\left[u_{n}\right], \nu\left(u_{n}\right)\right) d \mathcal{H}^{N-1},\right. \\
\left.u_{n} \stackrel{L^{1}}{\longrightarrow} g, \nabla u_{n} \stackrel{L^{1}}{\longrightarrow} G\right\}
\end{array}
$$


and

$$
\begin{aligned}
I_{2}(G)=\inf _{\left\{v_{n}\right\} \subset S B V\left(\Omega ; \mathbb{R}^{d \times N}\right)} & \left\{\liminf _{n \rightarrow \infty} \int_{\Omega} W\left(v_{n}, \nabla v_{n}\right) d x\right. \\
& \left.+\int_{S_{v_{n}}} \Psi_{2}\left(\left[v_{n}\right], \nu\left(v_{n}\right)\right) d \mathcal{H}^{N-1}, v_{n} \stackrel{L^{1}}{\longrightarrow} G\right\} .
\end{aligned}
$$

Using a relaxation result in Bouchitté, Fonseca \& Mascarenhas [8] for the energy $I_{2}(g, G)$, and a sequential characterization of the energy $I_{1}(g, G)$ together with Reshetnyak's Theorem (cf. Reshetnyak [26] ) and Alberti's Rank One Theorem (cf. Alberti [3]) it was then deduced that

$$
\begin{aligned}
I_{1}(g, G)=\int_{\Omega} W_{1}(G-\nabla g) d x+\int_{S_{g}} \gamma_{1}([g] & \left., \nu_{g}\right) d \mathcal{H}^{N-1} \\
& +\int_{\Omega} W_{1}\left(-\frac{d D^{c} g}{d\left|D^{c} g\right|}\right) d\left|D^{c} g\right|
\end{aligned}
$$

and

$$
\begin{aligned}
I_{2}(G)=\int_{\Omega} W_{2}(G, \nabla G) d x+\int_{S_{G}} \gamma_{2}\left(G^{+}, G^{-}, \nu_{G}\right) d \mathcal{H}^{N-1} \\
\quad+\int_{\Omega} W_{2}^{\infty}\left(G, \frac{d D^{c} G}{d\left|D^{c} G\right|}\right) d\left|D^{c} G\right| .
\end{aligned}
$$

We note here that the effect of the structured deformation is in this case captured in the first energy $I_{1}(g, G)$ through the limit energy density $W_{1}$ that depends on $G-\nabla g$.

Finally we remark that both integral representation results presented in this section refer to first-order structured deformations. A natural generalization, which will be the scope of a forthcoming work, will be to consider the same kind of integral representation problems in the context of secondorder structured deformations.

\section{References}

[1] Ambrosio L., A compactness theorem for a special class of functions of bounded variation, Boll. Un. Mat. Ital. 3-B (1989), 857-881.

[2] Alberti G., A Lusin type theorem for gradients, J. Funct. Anal. 100 (1991), 110-118.

[3] Alberti G., Rank-one property for derivatives of functions with bounded variation, Proc. Royal Soc. Edinburgh Sect. A bf 123 (1993), 237-274.

[4] Ambrosio L. and G. Dal Maso, On the representation in $B V\left(\Omega ; \mathbb{R}^{m}\right)$ of quasi-convex integrals, J. Funct. Anal. 109 (1992), 76-97. 
[5] Ambrosio L., N. Fusco and D. Pallara, Functions of Bounded Variation and Free Discontinuity Problems, Oxford University Press, 2000.

[6] Barroso A., G. Bouchitté, G. Buttazzo and I. Fonseca, Relaxation of bulk and interfacial energies, Arch. Rational Mech. Anal. 135 (1996), 107-173.

[7] Braides A. and A. Coscia, The interaction between bulk energy and surface energy in multiple integrals. Proc. Roy. Soc. Edinburgh Sect. A 124 (1994), No. 4, 737-756.

[8] Bouchitté G., I. Fonseca and L. Mascarenhas, A global method for relaxation, Arch. Rational Mech. Anal. 145 (1998), 51-98.

[9] Bouchitté G., I. Fonseca, G. Leoni and L. Mascarenhas, A global method for relaxation in $W^{1, p}$ and in $\mathrm{SBV}_{p}$, Arch. Ration. Mech. Anal. 165 (2002), No. 3, 187-242.

[10] Baía M., J. Matias and P. M. Santos, A relaxation result in the framework of structured deformations in the $B V$-setting, accepted for publication in Proc. Royal Soc. Edinburgh Sect. A.

[11] Choksi R., G. Del Piero, I. Fonseca and D. Owen, Structured deformations as energy minimizers in models of fracture and hysteresis, Mathematics and Mechanics of Solids 4 (1999), 321-356.

[12] Choksi R. and I. Fonseca, Bulk and interfacial energies for structured deformations of continua, Arch. Rational Mech. Anal. 138 (1997), 37-103.

[13] Carriero, M., A. Leaci and F. Tomarelli, A second order model in image segmentation: Blake and Zisserman functional, 25, Progress in Nonlinear Diff. Equations, (1996), 5772.

[14] Carriero M., A. Leaci and F. Tomarelli, Second order variational problems with free discontinuity and free gradient discontinuity, Calculus of Variations: Topics from the Mathematical Heritage of E. De Giorgi, Quad. Mat., 14, pp. 135-186. Dept. Math., Seconda Univ. Napoli, Caserta (2004).

[15] De Giorgi E. and L. Ambrosio, Un nuovo tipo di funzionale del calcolo delle variazioni, Atti Accad. Naz. Lincei, 82 (1988), 199-210.

[16] Del Piero G. and D. Owen, Structured deformations of continua, Arch. Rational Mech. Anal. 124 (1993), 99-155.

[17] Del Piero G. and D. Owen, Structured Deformations, Lecture Notes, XXII Scuola Estiva di Fisica Matematica, Ravello, September, 1997. Research Report No. 99-CNA-014, Center for Nonlinear analysis, Department of Mathematical Sciences, Carnegie Mellon University.

[18] Evans L. C. and R. F. Gariepy, Measure Theory and Fine Properties of Functions, Studies in Advanced Mathematics, CRC Press, 1992.

[19] Federer H., Geometric Measure Theory, Springer, Berlin, 1969.

[20] Fonseca I. and S. Müller, Quasi-convex integrands and lower semicontinuity in $L^{1}$. SIAM J. Math. Anal. 23 (1992), No. 5, 1081-1098

[21] Fonseca I. and S. Müller, Relaxation of quasiconvex functionals in $\mathrm{BV}\left(\Omega, R^{p}\right)$ for integrands $f(x, u, \nabla u)$. Arch. Rational Mech. Anal. 123 (1993), No. 1, 1-49

[22] Giusti E., Minimal Surfaces and Functions of Bounded Variation, Birkhäuser, 1984.

[23] Matias J. and P. M. Santos, A dimension reduction result in the framework of structured deformations, in preparation.

[24] Owen, D, Disarrangements in continua and the geometry of microstructure, K. Rajagopal ed., Recent advances in elasticity, viscoelasticity and inelasticity, World Scientific, 1995.

[25] Owen D. and R. Paroni, Second-order structured deformations, Arch. Rational Mech. Anal. 155 (2000), 215-235.

[26] Reshetnyak Y. G., Weak convergence of completely additive vector functions on a set, Siberian Math. J. 9(1968), 1039-1045 (translation of Sibirsk Mat. Z. 9 (1968), 13861394.)

[27] Ziemer W., Weakly Differentiable Functions, Springer-Verlag, 1989. 